\title{
Caracterización dasométrica \\ e índice de sitio en plantaciones de caoba en Tabasco, México
}

\author{
Dasometric characterization and site index in mahogany \\ plantations in Tabasco, Mexico
}

\section{Guadalupe Pérez González¹, Marivel Domínguez-Domínguez²*, Pablo Martínez-Zurimendi3,4 y Jorge D. Etchevers Barra5}

\section{RESUMEN}

\begin{abstract}
Se hizo una caracterización dasométrica y se determinó la calidad de sitio, en ocho plantaciones de Swietenia macrophylla King, en parcelas permanentes establecidas en el trópico de México. Las plantaciones estudiadas presentaban edades de 7 a 16 años, densidades de establecimiento de plantación

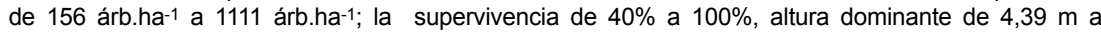
23,45 m, área basal de 2,3 $\mathrm{m}^{2}$ ha-1 a $15,7 \mathrm{~m}^{2} \mathrm{ha}-1$, volumen de madera con corteza de $14 \mathrm{~m}^{3} \mathrm{ha} \mathrm{a}^{-1} \mathrm{a}$ $185 \mathrm{~m}^{3} \mathrm{ha}^{-1}$, índice de Reineke de 176 a 505. Con base en la caracterización dasométrica se determinó que las plantaciones con mayor productividad se encuentran en la zona de La Chontalpa. Para determinar la calidad de sitio de estas plantaciones se probaron modelos de Bertalanffy, Chagoya, Logística, Monomolecular, Weibull, Korf y Wescom. El modelo de Korf presentó mejor coeficiente de determinación con alta confiabilidad para realizar la estimación de crecimiento en altura en árboles de la especie estudiada. Con la ecuación obtenida se determinó la evolución de la altura dominante con la edad y el índice de sitio para cada una de las plantaciones. Los índices de sitio para Swietenia macrophylla King en el estado de Tabasco se clasificaron como: baja $(11,5 \mathrm{~m})$, media $(14,5 \mathrm{~m})$ y alta $(17,5 \mathrm{~m})$ con edad base de 10 años. A partir de los resultados obtenidos, se puede considerar el potencial productivo de esta especie para futuros establecimientos en las zonas con mayores posibilidades.
\end{abstract}

PALABRAS CLAVE:

La Chontalpa, modelo de Korf, supervivencia, Swietenia macrophylla, Trópico de México.

\begin{abstract}
A dasometric characterization was made and the site quality was determined in eight mahogany plantations, in permanent plots established in the Tropical region of Mexico. Plantations studied were about 7 to 16 years old, starting densities of plantations from 156 to 1111 tree.ha- $^{-1}$; survival from $40 \%$ to $100 \%$, dominant height from $4,39 \mathrm{~m}$ to $23,45 \mathrm{~m}$, basal area from $2,3 \mathrm{~m}^{2}$ ha-1 to $15,7 \mathrm{~m}^{2} \mathrm{ha}^{-1}$, volume Colegio de Postgraduados. c.e.: lupi1904@hotmail.com

2* Colegio de Postgraduados, Campus Tabasco, Perif. Carlos A. Molina, km. 3.5, carr. Cárdenas-Huimanguillo, AP 24, CP 86500, H. Cárdenas, Tabasco, México, mdguez@colpos.mx Autor para correspondencia.

3 Sustainable Forest Management Research Institute UVa.INIA, ETS Ingenierías Agrarias, Universidad de Valladolid, Av. de Madrid núm. 57, 34007, Palencia, España.

4 Ecosur, Carretera Villahermosa-Reforma, km. 15.5, Ranchería Guineo, secc. II, CP 86280, Villahermosa, Tabasco. c.e.: pmartinez@ecosur.mx

5 Colegio de Postgraduados, Campus Montecillo, km. 36.5, carretera México-Texcoco, Montecillo, Estado de México, CP 56230. c.e.: jetchev@colpos.mx
\end{abstract}


of wood with bark from $14 \mathrm{~m}^{2} \mathrm{ha}^{-1}$ to $185 \mathrm{~m}^{3} \mathrm{ha}^{-1}$, Reineke index from 176 to 505. With the dasometric characterization basis, it was determined that plantations with increased productivity are located in the area of Chontalpa. To determine the quality of mahogany plantations areas, several models were tested: Bertalanffy, Chagoya, Logistics, Monomolecular, Weibull, Korf and Wescom. The Korf model resulted in a best fit with a probability of $(\alpha \leq 0,05)$. An equation was determined to evaluate the index site of mahogany, site index values for mahogany in the State of Tabasco were classified as: low $(11,5 \mathrm{~m})$, medium $(14,5 \mathrm{~m})$ and high $(17,5 \mathrm{~m})$ with a base of 10 year sold trees. With the basis of the obtained results, the potential productivity of this species may be considerer for future plantations in the areas with the greatest possibilities.

KEY WORDS:

La Chontalpa, Korf model, survival, Swietenia macrophylla, Tropic of Mexico.

\section{INTRODUCCIÓN}

Swietenia macrophylla King (caoba) y Cedrela odorata L. (cedro) son dos especies forestales del país que por su madera preciosa han sido sometidas a aprovechamiento intensivo, eso ha generado que se establezcan plantaciones para satisfacer la fuerte demanda de la industria maderera en México (Ramírez y Zepeda, 1994; Bravo, 2007). Las maderas preciosas, de estas y otras especies, apenas representan $0,5 \%$ de la producción maderable en México. Esta baja producción se ha debido a varios factores, entre ellos: la mini-fundización de las tierras que acompañó a los programas de colonización de los trópicos durante las décadas de los años sesenta y setenta del siglo pasado, los incendios y las plagas forestales que reducen la producción de madera. Todos estos factores han incidido en que los árboles de S. macrophylla y $C$. odorata sean escasos y de talla reducida (Challenger, 1998; Conafor, 2005). En México, a pesar de que $S$. macrophylla es una especie con alto valor económico, existe poca información cuanti- tativa acerca de su crecimiento, desarrollo y rendimiento, en plantaciones (García, 1998).

El éxito de una plantación forestal está fundamentado en el aumento en sus dimensiones: altura, diámetro, área basal y volumen maderable que determina su crecimiento en un periodo de tiempo determinado (Klepac, 1983). El manejo silvícola que se debe aplicar a las plantaciones forestales es importante para promover el crecimiento de los árboles y debe estar basado en mediciones dasométricas de la especie que consideran los incrementos en diámetro, altura y producción en volumen maderable. Los tratamientos silvícolas, tales como el aclareo, buscan mejorar la estructura del rodal y, lo más importante, incrementar el volumen útil en la corta final (Smith et al., 1997). En Tabasco se realizó un inventario forestal de las plantaciones de S. macrophylla, a partir del cual se generó una amplia base de datos que permitió analizar su crecimiento desde el año 2003 hasta el 2008. Con esa información se determinó el índice de sitio donde se desarrolla cada plantación.

\section{OBJETIVOS}

El presente trabajo tuvo como objetivo caracterizar dasométricamente altura, diámetro, área basal y volumen maderable de las plantaciones de Swietenia macrophylla King, en el estado de Tabasco, así como determinar el índice de sitio a través de un modelo de evolución de la altura dominante con la edad.

\section{METODOLOGÍA}

\section{Localización del área de estudio}

El estudio se realizó en el sureste de México, en el estado de Tabasco, en plan- 
taciones de S. macrophylla, en los municipios de Cunduacán, Huimanguillo y Centla, ubicados entre las coordenadas, $18^{\circ} 39^{\prime}$ y $17^{\circ} 15^{\prime}$ de latitud norte; y $91^{\circ} 00^{\prime}$ y $94^{\circ} 07^{\prime}$ de longitud oeste (INEGI, 2000; Fig. 1). De acuerdo con la clasificación de Köppen, modificada por García (1988), el clima para la zona de estudio es (Af) cálido húmedo con lluvias todo el año y (Am) cálido húmedo con abundantes lluvias en verano. La temperatura media anual mínima es $22,5^{\circ} \mathrm{C}$ y máxima de $30,5{ }^{\circ} \mathrm{C}$. El régimen de precipitaciones varía desde los $1947 \mathrm{~mm}$ a los $2290 \mathrm{~mm}$ anuales (INEGI, 2005). Los suelos predominantes en la zona de estudio son Fluvisol éutrico gléyico (Fleugl), Cambisol ferrali gléyico (CMflgl) y Gleysol mólico (GLmo) (Palma et al., 2008).

\section{Determinación de la caracterización dasométrica}

Para la realización de este estudio se dispuso de la información dasométrica consignada en el inventario de plantaciones de $S$. macrophylla en Tabasco, elaborado por la Comisión Estatal Forestal (Comesfor). Se analizaron datos de mediciones realizadas en los años 2003, 2004, 2005 y 2008, de la red de parcelas permanentes establecidas en ocho plantaciones de $S$. macrophylla, propiedad de los silvicultores.

Se midieron 21 parcelas permanentes, con 44 árboles en promedio, haciendo un total de 924 árboles. Los parámetros medidos fueron: la densidad

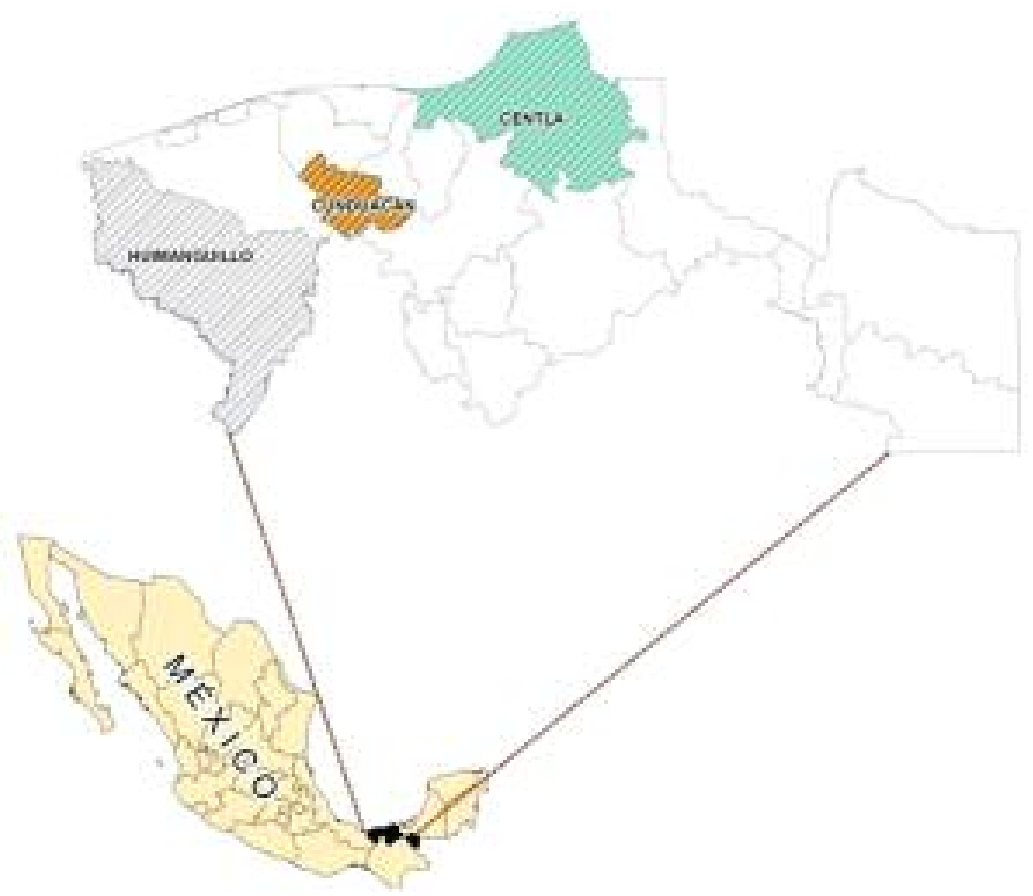

Figura 1. Localización de las plantaciones analizadas de S. macrophylla en Tabasco, México. 
del arbolado, la superficie de las parcelas, la edad (años) y la sobrevivencia de los árboles. Se midió el diámetro normal $(\mathrm{DBH})$ con una forcípula y la altura total de los árboles (HT) con una pistola Haga. Con los datos medidos en las parcelas permanentes se calcularon las siguientes variables: esbeltez, diámetro medio cuadrático, área basal, volumen de madera con corteza y árboles dominantes, de acuerdo con Assmann (1970). Se determinaron los índices de densidad del rodal de Reineke y el índice de Hart (Daniel et al., 1982). El índice de Reineke refleja la competencia entre los árboles y por tanto la disponibilidad de recursos que tienen los árboles para continuar creciendo adecuadamente. A partir de los datos dasométricos de las plantaciones y de las curvas de índice de sitio (como se explica más abajo en este apartado) se clasificó la calidad de cada una de las plantaciones estudiadas. Para la medición de la densidad de las plantaciones se utilizaron tres índices: área basal, índice de densidad de rodal de Reineke e índice de Hart-Becking.

\section{Área basal $(A B)$}

Se obtuvo a partir del diámetro a 1,3 $\mathrm{m}$ de altura como la sección transversal correspondiente. Se expresa en $\mathrm{m}^{2} \mathrm{ha}^{-1}$ (Avery y Burhart, 2002; Domínguez et al., 2006):

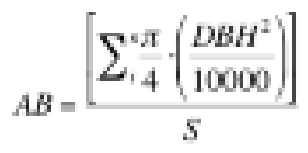

donde

$\mathrm{DBH}$ : diámetro a la altura del pecho en centímetros.

S: tamaño de la parcela en hectáreas.
Índice de densidad del rodal de Reineke (IDR)

Es un índice que expresa la medida cuantitativa de la densidad promedio que un rodal puede soportar, con referencia a un límite entre la relación del número de árboles por unidad de superficie y el tamaño promedio de los árboles para lograr un objetivo determinado; es independiente de la edad y de la calidad de sitio (Clutter et al., 1983). Se expresa:

$$
W R=N \cdot\left(\frac{D g}{25}\right)^{1, \text { tas }}
$$

donde

$\mathrm{N}$ : densidad de arbolado en árboles por hectárea.

Dg: diámetro medio cuadrático en centímetros.

El índice determina el número de árboles que existen en un rodal a través del estudio de un rodal puro, bien poblado y de edad uniforme con un diámetro medio cuadrático de $25 \mathrm{~cm}$ (Daniel et al., 1982). Para determinar el índice del rodal, Reineke (1933) generó una ecuación que al graficarla representa un valor de la pendiente igual a 1,605 , el cual se ha considerado como coeficiente general para las especies. Para la interpretación del IDR, Long (1985) define $60 \%$ de IDR máximo como el inicio de autoaclareo y $35 \%$ como límite de ocupación completo del sitio o estación.

\section{El índice de Hart-Becking}

Se define como la relación entre el espaciamiento medio del arbolado, calculado a partir de la densidad (N) y su altura dominante $(\mathrm{Ad})$, expresada en porcentaje (Codina, 2003).

$$
H=\frac{10000}{A d^{*} \sqrt{N}}
$$


donde

$\mathrm{H}$ : índice de Hart-Becking en porcentaje.

Ad: altura dominante en metros.

$\mathrm{N}$ : densidad de arbolado en árboles por hectárea.

\section{Análisis estadístico de la caracterización dasométrica}

Se realizó un análisis estadístico de correlación para establecer el grado de asociación entre las variables dasométricas y seleccionar las variables a utilizar en los modelos en la determinación del índice de sitio. De las variables que presentaron alta correlación se seleccionaron las que mejor se ajustaban en el modelo. También se realizó un análisis de varianza y una prueba de comparación de medias mediante Student-Newman-Keuls con $\alpha=0,05$ de las variables dasométricas, diámetro a la altura del pecho, diámetro medio cuadrático, altura total, esbeltez, área basal y volumen maderable con corteza.

\section{Determinación del índice de sitio}

Métodos para construir familias de curvas de índice de sitio

El índice de sitio es una representación gráfica que describe la relación altura dominante-edad de un rodal o árboles individuales y define el grado de productividad de un lugar. Para este estudio se aplicó el método de curva guía, el cual consiste en ajustar en forma analítica un modelo matemático para obtener una curva promedio o curva guía en todo el intervalo de observaciones de altura dominante-edad, a partir de la cual se trazaron curvas proporcionales, arriba y debajo de la curva guía, las cuales representan diferentes índices de sitio (Clutter et al., 1983).
Los modelos usados para determinar el índice de sitio en las plantaciones de $S$. macrophylla se generaron de acuerdo con los modelos matemáticos, usando la altura dominante y la edad de las plantaciones. La familia de curvas que se usó para el modelo, que mejor ajustó, fue la familia anamórfica. Lo anterior se debió a que estas curvas se caracterizan por presentar la misma forma, son proporcionales entre sí y la pendiente de la curva a cualquier edad guarda una relación constante entre ellas (Clutter et al., 1983). El punto de inflexión se presentó a la misma edad, ajustando de una forma analítica un modelo matemático para obtener una curva de guía para las observaciones de altura dominante y edad.

Se eligió como edad base 10 años, considerando los estudios de García (1998), Wescom (1979), Mayhew y Newton (1998), Lugo et al. (2003), para plantaciones de $S$. macrophylla. La edad base se seleccionó en función de dos criterios: estar cercano a la mitad del turno de corta y estar dentro del rango de edades medidas. Se calcularon los ajustes de las curvas de Von Bertalanffy, Chagoya, Logística, Monomolecular, Weibull, Korf y Wescom, con la base de datos de las plantaciones de S. macrophylla en Tabasco (Tabla 1).

Se probaron siete modelos con los crecimientos de las plantaciones, considerando las familias de curvas de índice de sitio propuestas por García (1998) en Quintana Roo, Wescom (1979) en Fiji, Mayhew y Newton (1998) en Sri Lanka y Vallejos (1996) en Colombia, Costa Rica, Honduras, Nicaragua y Panamá, por su similitud climática con Tabasco y las condiciones de cálculo similares a las referidas en este estudio. 
Tabla 1. Modelos usados para describir el crecimiento de las plantaciones de S. macrophylla.

\begin{tabular}{ll}
\hline Modelo & Ecuación \\
\hline Von Bertalanffy & $\mathrm{Ad}=\mathrm{A} 0^{*}\left(1-\mathrm{EXP}\left(-\mathrm{A} 1^{*} \mathrm{~T}\right)\right)^{\star *} 3$ \\
Chagoya & $\mathrm{Ad}=\mathrm{EXP}\left(\operatorname{LOG}(\mathrm{A} 0)-\mathrm{A} 1^{*}((1 / \mathrm{T})-0,1)\right)$ \\
Monomolecular & $\mathrm{Ad}=\mathrm{A} 0^{*}\left(1-\mathrm{A} 1^{*} \mathrm{EXP}\left(-\mathrm{A} 2^{*} \mathrm{~T}\right)\right)$ \\
Logístico & $\mathrm{Ad}=\mathrm{A} 0 /\left(1+\mathrm{A} 1^{*} \mathrm{EXP}\left(-\mathrm{A} 2^{*} \mathrm{~T}\right)\right)$ \\
Weibull & $\mathrm{Ad}=\mathrm{A} 0^{*}\left(1-\mathrm{EXP}\left(-\mathrm{A} 1^{*}\left(\mathrm{~T}^{* *} \mathrm{~A} 2\right)\right)\right)$ \\
Korf & $\mathrm{Ad}=\mathrm{A} 0^{*} \operatorname{EXP}\left(-\mathrm{A} 1^{*} \mathrm{~T}^{* *}-\mathrm{A} 2\right)$ \\
Wescom & $\mathrm{Ad}=\mathrm{A} 0^{*}\left(10^{* *}\left(-\mathrm{A} 1^{*}((1 / \mathrm{T}-0,1))\right)\right.$ \\
\hline
\end{tabular}

NотA: $\mathrm{Ad}=$ Altura dominante; $\mathrm{A} 0, \mathrm{~A} 1, \mathrm{~A} 2, \mathrm{~A} 3$ = parámetros de tasas de crecimiento; $\mathrm{EXP}=$ exponencial de la base de los logaritmos naturales; $T=$ tiempo.

Análisis estadístico para determinar el índice de sitio

Se usaron $70 \%$ de los datos para el desarrollo de los modelos matemáticos. Con una base de 68 datos se corrieron los modelos de Von Bertalanffy, Chagoya, Monomolecular, Logístico, Weibull, Korf y Wescom, para determinar el índice de sitio. Se utilizaron $30 \%$ de los datos para medir la bondad de los ajustes de una forma independiente, a través de los índices para la validación de los modelos (Huang et al., 2003; Domínguez et al., 2006); se estudiaron los errores a través de los parámetros MRES (error medio del modelo) y AMRES (diferencia absoluta media); también se obtuvo la eficiencia de cada modelo y el coeficiente de determinación ajustado.

\section{Procedimiento para la validación de los modelos}

$$
\text { MRFS }=\frac{\sum\left(y_{i}-\hat{y}_{i}\right)}{n}
$$

$\operatorname{MRES}(\%)=100 \cdot M R E S / \bar{y}$

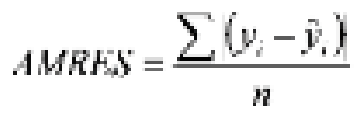

$\operatorname{AMRES}(\%)=100 \cdot A M R E S / \bar{y}$

donde

MRES: error medio del modelo.

AMRES: diferencia media absoluta.

$y_{i}$ : valor observado o real.

$\hat{y}_{i}: \quad$ valor estimado con el modelo.

$n$ : $\quad$ número de observaciones en la validación de los modelos.

$\bar{y}_{i}$ : $\quad$ valor promedio de los valores observados o reales. 
Los modelos se validaron aplicando las fórmulas de eficiencia del modelo (EF) que se considera un estimador para evaluar los modelos aplicados con regresiones no lineales (Huang et al., 2003).

$$
E F=1-\frac{\sum\left(y_{t}-\bar{y}_{4}\right)^{2}}{\sum\left(y_{t}-\bar{y}_{4}\right)^{2}}
$$

donde

$y_{i}$ : valor observado o real.

$\hat{y}_{i}$ : valor estimado con el modelo.

$\bar{y}_{i}$ : valor promedio de los valores observados o reales.

El coeficiente de determinación ajustado $\left(R^{2}\right.$ ajust $)$ no puede ser directamente obtenido en el análisis de varianza de la regresión no lineal, como medida de la bondad del ajuste, por lo que se hace una aproximación de la estimación del coeficiente de determinación ajustado, a través de la diferencia de la suma de cuadrados total y la suma de cuadrados medios de los residuos, para compararlo con la eficiencia del modelo (Domínguez et al., 2006).

$$
A p r o x-R^{2}{ }_{4 \times 1}=\frac{S S C-S S R}{S S C}
$$

donde

SSC: suma de cuadrado total.

SSR: suma de cuadrados medios de los residuos.

\section{RESULTADOS}

\section{Caracterización dasométrica}

Se evaluaron ocho plantaciones de Swietenia macrophylla King, siete de ellas ubicadas en los municipios de Cunduacán y Huimanguillo, en el área de La Chontalpa y una en el municipio de Centla, en el área de la costa del estado de Tabasco. Las plantaciones evaluadas se encuentran en un intervalo de edad de 7 a 16 años.

Los árboles de esas plantaciones fueron establecidos a diversos espaciamientos, existiendo una diversidad de densidades que va de 156 árb.ha-1 a 1111 árb.ha-1, ésta última establecida a una distancia corta de $3 \mathrm{~m} \times 3 \mathrm{~m}$ entre los árboles. Solamente dos plantaciones alcanzaron la máxima supervivencia, de $100 \%$, las demás plantaciones presentaron supervivencias entre $40 \%$ y $90 \%$ (Tabla 2).

La altura dominante como variable indicadora del índice de sitio en las plantaciones de $S$. macrophylla de mayor tamaño fue de $23 \mathrm{~m}$, a una edad de 16 años, y de $8 \mathrm{~m}$ a $17 \mathrm{~m}$, en las demás plantaciones a edades que fluctuaban entre 7 y 13 años. Las plantaciones presentaron un área basal máxima de $16 \mathrm{~m}^{2} \mathrm{ha}^{-1}$ y la mínima fue de $4 \mathrm{~m}^{2} \mathrm{ha}-1$. El máximo volumen maderable que presentaron las plantaciones fue $185 \mathrm{~m}^{3} \mathrm{ha}^{-1}$, y el mínimo volumen maderable fue de $14 \mathrm{~m}^{3} \mathrm{ha}^{-1}$ (Tabla 2).

A continuación se muestra la comparación de medias de Student-NewmanKeuls, de las variables dasométricas en que se realizó esta prueba estadística. Existen plantaciones donde los árboles tienen la misma edad, pero su crecimiento del diámetro a la altura del pecho es diferente, como el caso de tres plantaciones de 9 años que presentaron diámetros de $10 \mathrm{~cm}, 13 \mathrm{~cm}$ y $15 \mathrm{~cm}$. Las dos primeras no difirieron estadísticamente entre sí, pero el diámetro promedio de la tercera fue diferente de las anteriores, aún siendo de la misma edad. La plantación de mayor edad (16 años) presentó 
Tabla 2. Valores promedios de las características dasométricas de las plantaciones de S. macrophylla en Tabasco.

\begin{tabular}{lccccccc}
\hline Clave & Municipio & $\begin{array}{c}\text { Edad } \\
(\text { años })\end{array}$ & $\begin{array}{c}N \\
(a ́ r b . h a-1)\end{array}$ & $\begin{array}{c}S \\
(\%)\end{array}$ & $\begin{array}{c}A d \\
(m)\end{array}$ & $\begin{array}{c}A B \\
\left(m^{2} h a^{-1}\right)\end{array}$ & $\begin{array}{c}V \\
\left(m^{3} h a^{-1}\right)\end{array}$ \\
\hline $27-036$ & Cunduacán & 7 & 612 & 100 & 12 & 13 & 73 \\
$27-013$ & Centla & 9 & 206 & 40 & 12 & 7 & 14 \\
$27-039$ & Cunduacán & 9 & 556 & 70 & 8 & 8 & 36 \\
$27-041$ & Cunduacán & 9 & 944 & 60 & 15 & 13 & 89 \\
$27-048$ & Cunduacán & 11 & 121 & 60 & 16 & 7 & 54 \\
$27-034$ & Cunduacán & 13 & 156 & 100 & 14 & 4 & 37 \\
$27-053$ & Huimanguillo & 13 & 800 & 70 & 17 & 13 & 98 \\
$27-037$ & Cunduacán & 16 & 354 & 90 & 23 & 16 & 185 \\
\hline
\end{tabular}

NотA: $\mathrm{N}=$ densidad de arbolado; $\mathrm{S}=$ supervivencia; $\mathrm{Ad}=$ altura dominante; $\mathrm{AB}=$ área basal; $\mathrm{V}=$ volumen.

un diámetro a la altura del pecho de $23 \mathrm{~cm}$. Se esperaría que la plantación establecida a una densidad menor (625 árb.ha-1) (27-013) presentase un crecimiento mayor a las otras plantaciones con densidad de 1111 árb.ha-1 , por disponer éstos de más espacio entre individuos; sin embargo, resultó que esta plantación presentó el menor diámetro promedio de las tres, lo cual puede explicarse ya que las condiciones del sitio desempeñan un papel importante en el crecimiento de los árboles. En estas tres plantaciones se presentó una respuesta similar en el crecimiento de la altura total del árbol. La plantación que mostró el menor crecimiento en diámetro y en altura total, presentó el mayor índice de esbeltez, de 97\% (Tabla 3).

La mayoría de las plantaciones presentaron índices de esbeltez menores

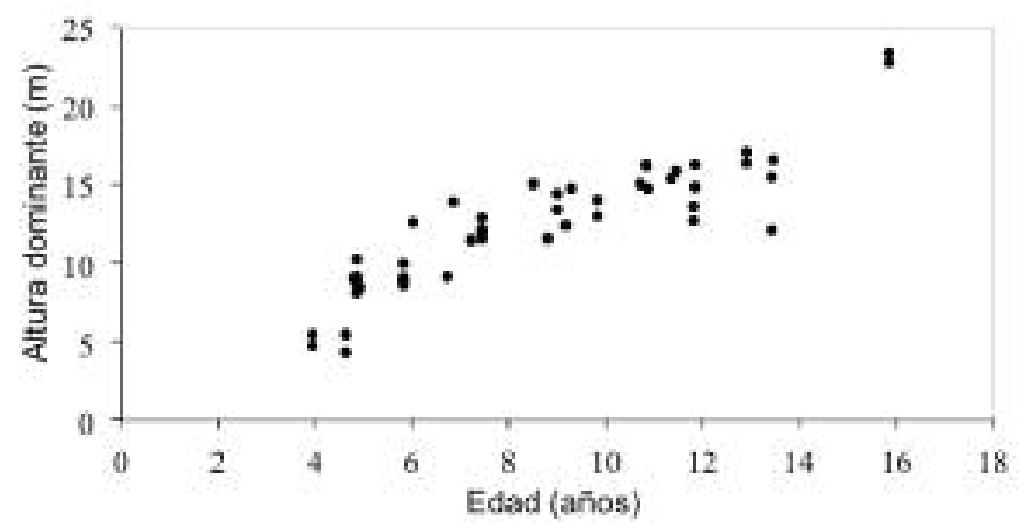

Figura 2. Altura dominante en plantaciones de S. macrophylla en Tabasco, México. 
Tabla 3. Comparación de medias de las variables dasométricas en plantaciones de S. macrophylla en Tabasco.

\begin{tabular}{lcccccc}
\hline Clave & $\begin{array}{c}\text { Edad } \\
\text { (años) }\end{array}$ & $\begin{array}{c}D B H \\
(\mathrm{~cm})\end{array}$ & $\begin{array}{c}H T \\
(\mathrm{~m})\end{array}$ & $\begin{array}{c}\text { Esbeltez } \\
(\%)\end{array}$ & $\begin{array}{c}A B \\
\left(m^{2} h a-1\right)\end{array}$ & $\begin{array}{c}V \\
\left(m^{3} a ́ r b-1\right)\end{array}$ \\
\hline $27-036$ & 7 & $15 \mathrm{ed}$ & $11 \mathrm{~d}$ & $76 \mathrm{~b}$ & $0,018 \mathrm{~d}$ & $0,107 \mathrm{ed}$ \\
$27-039$ & 9 & $13 \mathrm{~d}$ & $9 \mathrm{e}$ & $77 \mathrm{~b}$ & $0,014 \mathrm{ed}$ & $0,072 \mathrm{ed}$ \\
$27-013$ & 9 & $10 \mathrm{f}$ & $9 \mathrm{e}$ & $97 \mathrm{a}$ & $0,011 \mathrm{e}$ & $0,065 \mathrm{e}$ \\
$27-041$ & 9 & $15 \mathrm{~d}$ & $13 \mathrm{c}$ & $90 \mathrm{a}$ & $0,020 \mathrm{~d}$ & $0,143 \mathrm{~d}$ \\
$27-048$ & 11 & $21 \mathrm{~b}$ & $15 \mathrm{~b}$ & $61 \mathrm{c}$ & $0,056 \mathrm{a}$ & $0,443 \mathrm{~b}$ \\
$27-034$ & 13 & $19 \mathrm{c}$ & $12 \mathrm{c}$ & $70 \mathrm{~b}$ & $0,033 \mathrm{c}$ & $0,236 \mathrm{c}$ \\
$27-053$ & 13 & $14 \mathrm{e}$ & $13 \mathrm{c}$ & $97 \mathrm{a}$ & $0,016 \mathrm{ed}$ & $0,121 \mathrm{ed}$ \\
$27-037$ & 16 & $23^{\mathrm{a}}$ & $21 \mathrm{a}$ & $94 \mathrm{a}$ & $0,045 \mathrm{~b}$ & $0,504 \mathrm{a}$ \\
\hline
\end{tabular}

NоTA: $\mathrm{DBH}=$ diámetro a la altura del pecho; $\mathrm{HT}=$ altura total; $\mathrm{AB}=$ área basal; $\mathrm{V}=$ volumen maderable; árb = árbol. Media con las mismas letras son estadísticamente iguales con $\alpha: 0,05$ según la prueba de medias Student-Newman-Keuls

de $80 \%$, las cuales, según González (2001), serían estables; los índices mayores de $81 \%$ serían inestables y los mayores de $100 \%$ de alta inestabilidad. La esbeltez, en general, refleja el vigor del árbol, ésta varía con la edad: esbeltez de hasta $100 \%$ es normal en la fase juvenil de la planta, a partir de los 10 años se espera que la esbeltez sea inferior a $80 \%$; lo contrario es síntoma de árboles muy finos que pueden quebrarse fácilmente.

La máxima área basal de los árboles la presentó la plantación de 11 años de edad, a diferencia de la plantación de 16 años de la zona de La Chontalpa, que presentó menor área basal. En general el volumen de madera por árbol de S. macrophylla fue similar en todos los sitios; la mayoría de las plantaciones no presentaron diferencias cuando se practicó el análisis de comparación de medias. La excepción la constituye una plantación de 11 años con densidad de 156 árb.ha-1 $^{-1}$ y otra de 16 años con densidad de 400 árb.ha-1, establecidas en la misma zona de La Chontalpa.
El máximo volumen maderable por árbol lo presentó la plantación 27-037 con

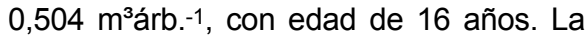
plantación que presentó el mínimo volumen maderable fue la 27-013 con $0,065 \mathrm{~m}^{3}$ árb.-1, con edad de 9 años. La figura 2 muestra la relación entre la edad de las plantaciones y la altura dominante y provee información acerca de la productividad futura de las plantaciones de la zona de Tabasco. La densidad máxima de árboles obtenida en las plantaciones en desarrollo de $S$. macrophylla fue de 1000 árb.ha- $^{-1}$, pero el volumen maderable en este caso fue de sólo $0,05 \mathrm{~m}^{3} a ́ r b .-1$, la mínima densidad de las plantaciones fue

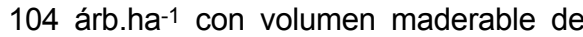
0,39 $\mathrm{m}^{3}$ árb.-1. Las plantaciones que tenían

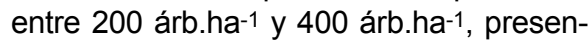
taron volumen maderable hasta de 0,5 mª́rb.-1; en cambio las plantaciones con densidades entre 600 árb.ha-1 y 1000 árb.ha-1, sólo alcanzaron $0,03 \mathrm{~m}^{3}$ árb.-1 (Fig. 3). Se puede ver claramente el efecto de la densidad del arbolado en la producción de volumen maderable medio. Esta misma relación se obtuvo con el área basal y la densidad. El área basal deter- 


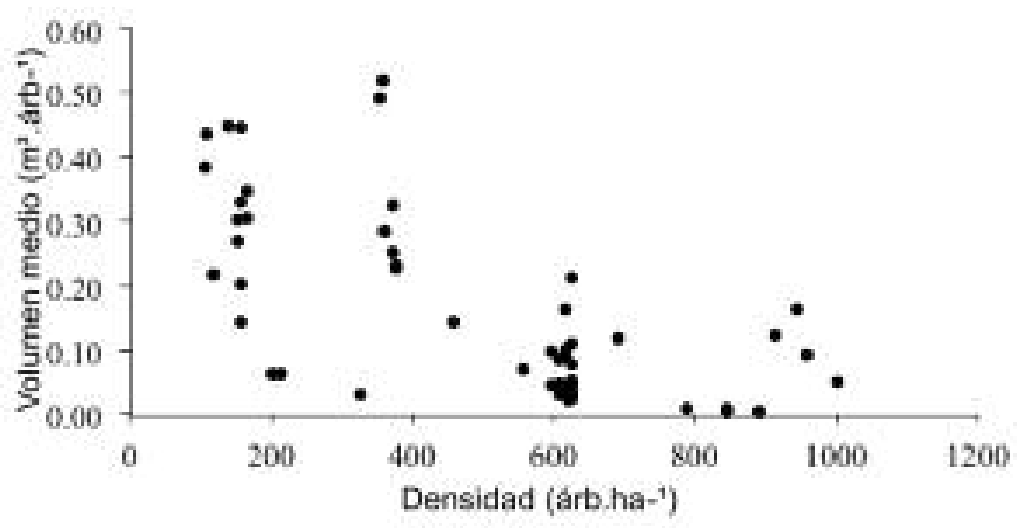

Figura 3. Representación del volumen maderable medio a diferentes densidades de arbolado, en plantaciones de S. macrophylla en Tabasco, México.

mina la competencia por espacio, por lo tanto cuando se tiene mayor número de árboles en una hectárea, el área basal por árbol disminuye drásticamente.

El índice máximo de densidad del rodal de Reineke en las plantaciones fue de 505. De acuerdo con Long (1985) una de ellas está en plena ocupación de sitio, cuatro plantaciones están en etapa de crecimiento (Fig. 4) y tres han entrado en etapa de autoaclareo o muerte por selección natural. En las plantaciones que presentaron IDR hasta 176 se encuentran los árboles en crecimiento; IDR mayor de 176 hasta 303 se encuentra en plena ocupacion de sitio, IDR mayor de 303 hasta 505 han entrado a la fase de autoaclareo de los árboles de forma natural, esto debido al exceso de competencia por espacio, luz y nutrimentos.

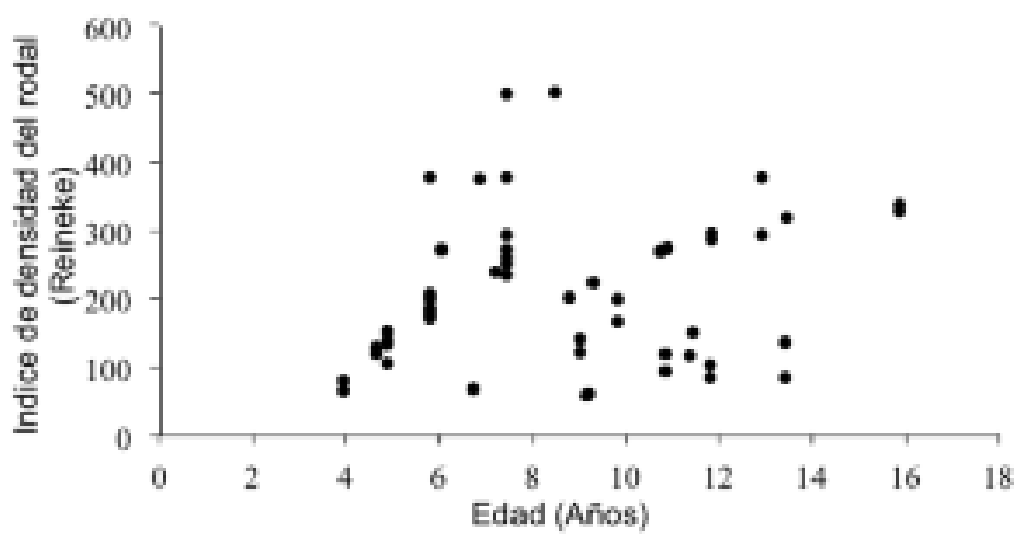

Figura 4. Índice de densidad del rodal de Reineke en plantaciones de S. macrophylla, de acuerdo con Long (1985). 


\section{Índice de sitio}

De los siete modelos probados (Bertalanffy, Chagoya, Logístico, Monomolecular, Weibull, Korf y Wescom) para determinar el índice de sitio en las plantaciones de $S$. macrophylla, el análisis de regresión no lineal aplicado demostró que el modelo de Korf es estadísticamente diferente, como se muestra en la tabla 4. A continuación se presentan los resultados de los valores del coeficiente de determinación ajustado y la eficiencia de los modelos que determinó la selección del modelo (Tabla 5). De los siete modelos, el que mejor eficiencia presentó fue el de Korf, así como el mejor coeficiente de determinación ajustado $(0,80$ en ambos casos). Los modelos de Von Bertalanffy y Chagoya resultaron muy bajos y negativos en la eficiencia, por el bajo ajuste de esos modelos. De los siete modelos probados para construir la curva de índice de sitio, la curva guía con el modelo de Korf fue la que mejor ajuste presentó y mayor eficiencia del modelo para las plantaciones estudiadas, siendo 12,34 m la altura dominante a una edad base de 10 años. El modelo de Korf quedó como sigue:

$$
A d=12,34 \cdot e^{-4,9(t)^{-0,29}}
$$

donde

Ad: la altura dominante.

e: la base de los logaritmos naturales.

$\mathrm{t}$ : tiempo.

Posteriormente, con la curva guía del modelo de Korf seleccionado, se determinaron tres índices de sitio, con tres curvas de calidad, las cuales se clasificaron como baja, media y alta. El índice de sitio de las plantaciones que presentaron una productividad alta fue de $17,5 \mathrm{~m}$; las plantaciones que presentaron una productividad media tuvieron un índice de sitio de $14,5 \mathrm{~m}$ y las que presentaron una productividad baja 11,5 m (Fig. 5).

Tabla 4. Resultados de análisis de regresión no lineal y parámetros estimados para el modelo de altura dominante de Korf en S. macrophylla.

\begin{tabular}{lccc}
\hline Fuente & G.L & Suma de cuadrados & Cuadrados medios \\
\hline Regresión & 3 & 4954,8 & 1651,6 \\
Residual & 25 & 109 & 4,3588 \\
Total no corridos & 28 & 5063,8 & \\
Total corridos & 27 & 560,4 & \\
\hline & & & \\
Variable & Estimación & Intervalo de confianza al 95\% & Superior \\
& & Inferior & 60,060 \\
& & & 4,934 \\
Término independiente & 21426,4 & -43948 & 128 \\
A0 & 180,1 & $-118,8$ & 2,2146 \\
A1 & 4,9 & $-1,6326$ & \\
A2 & 0,291 &
\end{tabular}


Tabla 5. Eficiencia y $\mathrm{R}^{2}$ ajustada de los modelos de altura dominante en S. macrophylla.

\begin{tabular}{lcc}
\hline Modelos & $R^{2}$ ajustada & Eficiencia $(E F)$ \\
\hline Von Bertalanffy & 0,7655 & $-5,08$ \\
Chagoya & 0,7890 & $-2,33$ \\
Monomolecular & 0,7749 & 0,77 \\
Logístico & 0,7962 & 0,79 \\
Weibull & 0,7731 & 0,76 \\
Korf & 0,8015 & 0,80 \\
Wescom & 0,7890 & 0,79 \\
\hline
\end{tabular}

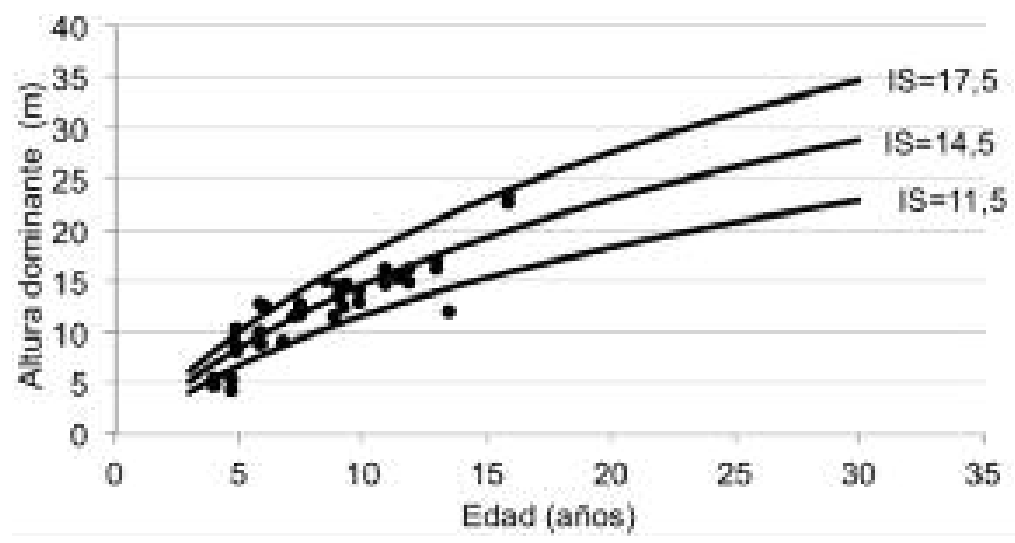

Figura 5. Calidades de sitio para plantaciones de S. macrophylla en Tabasco, México.

\section{DISCUSIÓN}

El análisis estadístico de los datos de las plantaciones de Swietenia macrophylla King, permitió caracterizar el crecimiento de la especie, observándose que existe poca variabilidad en diámetro a la altura del pecho, altura total, edad, área basal y volumen. Las plantaciones forestales tropicales están altamente influidas por la densidad y son afectadas en el crecimiento, principalmente, debido a que los árboles requieren mucho espacio e iluminación, sobre todo tratándose de especies perennes (Dawkins, 1961). El factor densidad fue relevante para la productividad de las plantaciones en estudio; en aquellas plantaciones que presentaron más de 1000 árb.ha-1 el volumen maderable fue de $0,05 \mathrm{~m}^{3}$ árb. ${ }^{-1}$, mientras que en la plantación con densidad de 104 árb.ha-1 el volumen fue de $0,39 \mathrm{~m}^{3}$ árb.-1. Lo anterior indica que la densidad es un factor que afecta drásticamente el volumen o 
producto final, como se ha demostrado en estudios en Puerto Rico, Martinica, Filipinas, México, Indonesia y Sri Lanka, esta última con espaciamientos de $2 \mathrm{~m} x$ $2 m$ y $3 m \times 3 m$ (Mayhew y Newton, 1998).

Otros factores que afectan a las plantaciones son las diferencias en las condiciones de sitio, éstos podrían alterar la pendiente de la línea de máxima densidad en la gráfica de poblaciones monoespecíficas (Lonsdale y Watkinson, 1983), además de los factores físicos como el viento y las plagas forestales que ocasionan daño mecánico o fisiológico al árbol que disminuye la densidad (Conafor, 2005). La densidad es un factor que influye fuertemente en el crecimiento de las plantaciones de $S$. macrophylla, al cual se debe prestar atención en los primeros 5 años de establecimiento de una plantación (Mayhew y Newton, 1998).

El área basal determinada en las plantaciones de $S$. macrophylla fue de 16,4 $\mathrm{m}^{2} \mathrm{ha}^{-1}$ como máximo y $4 \mathrm{~m}^{2} \mathrm{ha}^{-1}$ como mínimo; la mayor área basal detectada a la edad de 7 años fue $13 \mathrm{~m}^{2} \mathrm{ha}^{-1}$. Este último resultado supera el de otro estudio con el mismo manejo silvicola, en la misma especie y edad, en que el área basal fue de $10 \mathrm{~m}^{2} \mathrm{ha}^{-1}$ (Bauer, 1987). En el caso de plantaciones en Honduras (AFE-COHDEFOR, 1989), se ha determinado que plantaciones con área basal de más de $25 \mathrm{~m}^{2} \mathrm{ha}^{-1}$ requieren un tratamiento de aclareo con intensidad de $35 \%$, de $21 \mathrm{~m}^{2}$ ha-1 a 24,9 m²ha-1 la intensidad sugerida es de $30 \%$, buscando disminuirla hasta los $16 \mathrm{~m}^{2} \mathrm{ha}^{-1}$. Las plantaciones en Tabasco están en el rango sugerido por la AFE-COHDEFOR (1989); las cuales con un buen manejo podrían presentar mayor potencial de producción.

El índice de rodal de Reineke expresa el número de árboles por hectárea con un diámetro de $25 \mathrm{~cm}$; al aplicar este índice se determinó que las plantaciones de $S$. macrophylla presentaron un IDR 505. De acuerdo con la interpretación de Long (1985), se determinó que tres plantaciones han entrado en autoaclareo porque tienen más de $60 \%$ de IDR, una plantación está en etapa de máxima ocupación del sitio con más de $35 \%$ de IDR y cuatro plantaciones presentan árboles en crecimiento. Los límites de 35\% a 60\% del IDR definen el intervalo de densidad dentro del cual la productividad es máxima (Langsaeter, 1941). Este índice se ha determinado en otras especies como Cedrela odorata, con 588 de IDR como valor máximo y en Tectona grandis, con 1100 como máximo IDR, con árboles en plena ocupacion de sitio que ya requieren aclareos (Jerez et al., 2003; Murillo, 2008).

En cuanto al diámetro de los árboles en las plantaciones de $S$. macrophylla en Tabasco, se vio afectado por la densidad y el sitio; sin embargo, los resultados de este estudio concuerdan con los de otros realizados en Filipinas, donde se ha demostrado que la especie llega a alcanzar 1,8 $\mathrm{m}$ de altura en el primer año y de $15 \mathrm{~cm}$ a $20 \mathrm{~cm}$ de diámetro en 14 años. En lquitos, Perú, alcanza los $27 \mathrm{~cm}$ de diámetro y de $20 \mathrm{~m}$ a $25 \mathrm{~m}$ de altura en 12 años (Conabio, 2001), bajo manejo silvícola. Sin embargo, el diámetro de cualquier especie es altamente sensible a los cambios con la densidad (Gadow et al., 2007). En general, el diámetro crecerá con base en el manejo que se aplique a dicha especie y la productividad del sitio, además de considerar otros factores como la densidad de plantación, el tamaño de la copa, la presencia de enfermedades, la aplicación de fertilizante, las características genéticas, entre otros.

De las ocho plantaciones estudiadas, 50\% presentó índices de esbeltez entre $90 \%$ y $97 \%$, otro $50 \%$ presentó índices entre $61 \%$ y $77 \%$. El índice de esbeltez es la relación entre la altura y el 
diámetro normal; es un valor que ha sido utilizado como un indicador de la estabilidad de los árboles contra daños ocasionados por fuerzas mecánicas; valores bajos de esbeltez están asociados con árboles más cónicos que pueden ser más resistentes al efecto de fuertes vientos (Arias, 2004). Entre más alto sea el valor de esbeltez, menos estable es el árbol ante los daños mecánicos (Durlo y Denardi, 1998).

La supervivencia de los árboles fue superior a $60 \%$ en siete de las plantaciones estudiadas con un espaciamiento de $3 \mathrm{~m} \times 3 \mathrm{~m}$ hasta $8 \mathrm{~m} \times 8 \mathrm{~m}$ y en la plantación restante fue de $40 \%$. Estudios anteriores en plantaciones de Cedrela odorata en Tabasco indican $71 \%$ a $97 \%$ de supervivencia (Murillo, 2008). Haggar et al. (2000), mencionan que $60 \%$ de supervivencia es considerado un porcentaje alto. La supervivencia puede ser afectada en gran medida por los espaciamientos en las especies tropicales (Evans y Turnbull, 2004), además, por ser un árbol perennifolio es más vunerable a la sequía y su lento crecimiento hace que esté expuesto a condiciones ambientales desfavorables (Barchuk y Díaz, 1999). Cabe señalar que, de acuerdo con la Comisión Nacional Forestal (Conafor, 2008), para que una plantación sea sujeto de apoyo debe cumplir con $70 \%$ a $80 \%$ de supervivencia a un año de edad. La supervivencia de la especie está más relacionada con las condiciones del sitio, con la cantidad de luz, agua y nutrimentos del suelo.

El volumen maderable es el producto final de toda plantación de S. macrophylla con fines comerciales. Resultados de Xue y Hagihara (2008), señalan que el efecto de la densidad sobre el crecimiento se ve reflejado en la altura, debido a que ésta se ve disminuida en su crecimiento con el aumento de la densidad y, por lo tanto, se ve afectado el volumen. Esto se debe principalmente a la competencia por espacio, luz, agua, nutrimentos y a características genéticas de la especie, plagas y enfermedades, huracanes, tipo de suelo, entre otros. El volumen maderable en las plantaciones de 7 años fue de $13 \mathrm{~m}^{3} \mathrm{ha}^{-1} \mathrm{y}$ en plantaciones de 16 años de edad de $185 \mathrm{~m}^{3} \mathrm{ha}^{-1}$. En otros estudios con esta misma especie, con manejo silvícola en Quintana Roo, reportaron volumen de madera de $22,8 \mathrm{~m}^{3} \mathrm{ha}^{-1}$ a edad de 5 años y 50,3 $\mathrm{m}^{3} \mathrm{ha}^{-1}$ a 15 años (García et al., 2007) y en la misma localidad García et al. (2011) predicen, mediante una modelización del crecimiento, un volumen de madera de $32 \mathrm{~m}^{3} \mathrm{ha}^{-1}$ a edad de 5 años y $140 \mathrm{~m}^{3} \mathrm{ha}^{-1}$ a 15 años. En Indonesia se obtienen crecimientos de $18 \mathrm{~m}^{3} \mathrm{ha}^{-1} \mathrm{año}^{-1}$ a la edad de 20 años, en la Isla de Fiji $5 \mathrm{~m}^{3} \mathrm{ha}^{-1}$ año-1 a edad de 35 años, en la Isla de Martinica $14 \mathrm{~m}^{3} \mathrm{ha}^{-1} \mathrm{año}^{-1}$ a $25 \mathrm{~m}^{3} \mathrm{ha}-1$ a edad de 35 años, en la Isla de Guadalupe $17 \mathrm{~m}^{3} \mathrm{ha}^{-1} \mathrm{añno}^{-1}$ a edad de 25 años y en Belice alcanza un crecimiento de $6 \mathrm{~m}^{3} \mathrm{ha}^{-1} \mathrm{añno}^{-1}$ totalizando un volumen de $280 \mathrm{~m}^{3} \mathrm{ha}^{-1}$ a edad de 50 años (Mayhew y Newton, 1998).

La determinación del índice de sitio en plantaciones de $S$. macrophylla a las cuales no se les ha dado manejo silvícola de aclareo, poda y fertilización, fueron clasificados en tres tipos: como bajo $(11,5 \mathrm{~m})$, medio $(14,5 \mathrm{~m})$ y alto $(17,5 \mathrm{~m})$ con una edad base de 10 años. García (1998) y García et al. (1998) determinaron cinco índices de sitio $(12,14,16,18$ y $20 \mathrm{~m}$ ) a una edad base de 30 años en plantaciones de S. macrophylla en Quintana Roo, con los modelos de Chapman y Richards y Weibull, los cuales sobreestiman las edades, sin embargo, esto es posible debido a los hábitos de crecimiento y edades de las plantaciones, además la consideran como una especie de lento crecimiento. En una buena calidad de sitio los turnos son más cortos que en los sitios de mediana y baja 
calidad (Assmann, 1961; Aguirre y Zepeda, 1984; Madrigal y Ramírez, 1995; Rodríguez, 1996). Wescom (1979) determinó seis índices de sitio con edad base de 10 años en $S$. macrophylla en Isla de Fiji; los índices de sitio que encontró fueron $(10,13,16,19,22$ y 25 m) y Rodríguez (1996) en Chetumal, Quintana Roo, determinó cuatro índices de sitio $(14,16$, 20 y $22 \mathrm{~m}$ ) en plantaciones de esta especie sin manejo silvícola a una edad base de 30 años.

En las plantaciones estudiadas los modelos con mejores ajustes fueron Bertalanffy, Chagoya, Logística, Monomolecular, Weibull, Korf y Wescom. El modelo de Weibull presentó un coeficiente de determinación de 0,77. Éste último es menor, comparado con otro estudio en plantaciones de Quintana Roo donde se encontró un R2 0,99 (García et al., 2007). El modelo Weibull es el más usado en las plantaciones de $S$. macrophylla, sin embargo, aquí el modelo fue menos eficiente; otros modelos usados para esta especie han sido Wescom en la Isla de Fiji, Schumacher en Quintana Roo y Chagoya en Costa Rica (Rodríguez, 1996; García et al., 1998).

\section{CONCLUSIÓN}

Este estudio permitió comprender el crecimiento de las plantaciones de Swietenia macrophylla King, en el estado de Tabasco. La caracterización dasométrica reveló que las plantaciones en desarrollo de S. macrophylla en Tabasco se encuentran en densidades de 100 árb.ha-1 a 1000 árb.ha-1 con edad de 4 a 16 años, con área basal entre $4 \mathrm{~m}^{2} \mathrm{ha}-1$ y $16 \mathrm{~m}^{2} \mathrm{ha}^{-1}$, con volúmenes que van desde los $14 \mathrm{~m}^{3} \mathrm{ha}^{-1}$ hasta los $185 \mathrm{~m}^{3} \mathrm{ha}^{-1}$. De acuerdo con el índice de densidad de rodal de Reineke, de las ocho plantaciones, cuatro están en estado de crecimiento, una en plena ocupación del sitio que necesita manejo silvícola de aclareo $y$ tres de ellas han entrado en autoaclareo, habiendo entrado los árboles en competencia y por tanto rebasado el momento de la aplicación de aclareo.

Para la determinación del índice de sitio los modelos de Wescom, Weibull, Logístico y Monomolecular presentaron buenos ajustes y eficiencias, sin embargo el modelo de Korf fue el mejor. Se definieron tres curvas de índice de sitio, determinando $11,5 \mathrm{~m}$ para el índice de sitio bajo, $14,5 \mathrm{~m}$ para índice de sitio medio y $17,5 \mathrm{~m}$ para el índice de sitio alto, todos ellos a edad base de 10 años. Las aportaciones de este trabajo en cuanto a la caracterización dasométrica y determinación de índice de sitio, servirán para proyectar la producción esperada y el manejo silvícola a aplicar en las plantaciones de $S$. macrophylla en Tabasco, aportando conocimiento de las zonas con mayor potencial productivo para el establecimiento de esta especie en la zona tropical de México.

\section{AGRADECIMIENTOS}

Esta investigación fue realizada con el apoyo financiero de la Fundación Produce, Tabasco, del proyecto "Diagnóstico del estado silvícola y desarrollo de un plan de aclareos en plantaciones de cedro y caoba en Tabasco".

\section{REFERENCIAS}

AFE-COHDEFOR. 1989. El sector forestal hondureño. Proyecto HON/ 88/003FAO/PNUD. Departamento de Planificación. Tegucigalpa, Honduras. 25 p.

Aguirre C., O.A. y E.M. Zepeda B. 1984. Estimación de índice de sitio para Pinus pseudostrobus Lindl., en la 
región de Iturbe, Nuevo León. Tesis profesional. Universidad Autónoma Chapingo. División de Ciencias Forestales. Chapingo, México. $71 \mathrm{p}$.

Arias, D. 2004. Estudio de las relaciones alturadiámetro para seis especies maderables utilizadas en programas de reforestación en la Zona Sur de Costa Rica. Revista Forestal Kurú 1(2):1-11.

Assmann, E. 1961. Waldertragskunde. Munich, Alemania, Bayerischer Landwirtschaftsverlag. $490 \mathrm{p}$.

Assmann, E. 1970. The principles of forest yield study. 1a. ed. Pergamon Press. Oxford. 506 p.

Avery, E.T. y E.H. Burkhart. 2002. Forest measurements. Fifth ed. McGraw-Hill. Nueva York, EUA. 456 p.

Barchuk, A.H. y M.P. Díaz. 1999. Regeneration and structure of Aspidosperma quebracho-blanco Schl. in the Arid Chaco (Córdoba, Argentina). Forest Ecology and Management 118:31-36.

Bauer, G.P. 1987. Swietenia macrophylla King and S. macrophylla x. S. mahogany development and growth: the nursery phase and the establishment phase in linne planting in the Caribbean National Forest, Puerto Rico. Tesis de Maestría, College of Environmental Science and forestry, Faculty of Forestry, State University of New York. EUA. 310 p.

Bravo M., A. 2007. Estimación maderable y evaluación financiera de plantaciones forestales comerciales de cedro y caoba en Oaxaca, México. Tesis de Maestría. Montecillo, Texcoco, Edo. de México. 86 p.

Challenger, A. 1998. Utilización y conservación de los ecosistemas terrestres de México: pasado, presente y futuro. Conabio, Insti- tuto de Ecología, UNAM y Agrupación

Sierra Madre S.C., México. s/p.

Clutter, J.L., J. Forston, L. Pienaar, G. Brister y R. Bailey. 1983. Timber management: a quantitative approach.Wiley. Nueva York, EUA. 333 p.

Codina C., J. 2003. El índice de Hartbecking y su justa medida. Foresta 24:50-52.

CONABIO (Comisión Nacional de la Biodiversidad). 2001. Árboles y arbustos nativos potencialmente valiosos para la restauración ecológica y la reforestación. Instituto de Ecología. Universidad Nacional Autónoma de México. México D. F. 236 p.

CONAFOR (Comisión Nacional Forestal). 2005. Informe de la situación del medio ambiente en México. Disponible en línea: http://148.223.105.188: 2222/ gif/snif_portal/option=com_contenttask=viewid=50\&ltemid=64/index.php. Consultada el 16 de julio de 2009.

CONAFOR (Comisión Nacional Forestal). 2008. Programa Forestal de la Comisión Estatal Forestal de Tabasco. Disponible en línea: www.conafor. gob.mx. Consultada el 23 de septiembre de 2009.

Daniel, T.W., J.A. Helms y F.S. Baker. 1982. Principios de silvicultura. (Trad. Ramón Elizondo Mata). McGraw-Hill. México D.F. 491 p.

Dawkins, H.C. 1961. Estimating total volumes of some Caribbean trees. Caribbean Forester 22(3-4):62-63.

Domínguez D., M., F. Bravo O. y M. Del Río G. 2006. Modelo de tamaño de copa en Pinus sylvestis L., en bosques del centro de España. Interciencia 31(3):168-175. 
Durlo, D.M. y L. Denardi. 1998. Morfometría de Cabralea canjerana, em mata Secundaria nativa do Rió Grande do Sul. Revista Ciência Florestal 1(8):55-66.

Evans, J. y J.W. Turnbull. 2004. Plantation Forestry in the Tropics: The Role, Silviculture, and Use of Planted Forests for Industrial, Social, Environmental, and Agroforestry Purposes. 3a. ed. Oxford University Press, Nueva York. 467 p.

Gadow, K.V., S. Sánchez O. y J. G. Álvarez G. 2007. Estructura y crecimiento del bosque. IUFRO World Series. 242 p.

García C., X. 1998. Predicción del rendimiento de Swietenia macrophylla King (caoba) en plantaciones forestales. Tesis de Maestría en Ciencias. Colegio de Postgraduados. Montecillo, México.114 p.

García C., X., H. Ramírez M., C. Rodríguez F., J. Jasso M. y C.A. Ortiz S. 1998. Índice de sitio para caoba (Swietenia macrophylla King) en Quintana Roo, México. Ciencia Forestal en México. 23(84):9-18.

García C., X., J. Flores G., y J. D. Benavides S. 2007. Índice de sitio para Cedrela odorata L. (Cedro rojo) en Quintana Roo, México. Ciencia Forestal en México 32(101):71-92.

García C., X., B. Rodríguez S. y J. Islas G. 2011. Evaluación financiera de plantaciones forestales de caoba en Quintana Roo. Revista Mexicana de Ciencias Forestales 2 (7):7-26.

García, E. 1988. Modificaciones al sistema de clasificación climática de Köppen. México. Universidad Autónoma de México, Instituto de Geografía. México D.F. 252 p.
González M., J.M 2001. Introducción a la silvicultura general. Universidad de León. Escuela Técnica Superior de Ingeniería Agraria. España. 271 p.

Haggar, J., K. Wightman, L. Sosa, K.H. Van, J.A. Contreras y G. Hernández. 2000. Una estrategia para hacer rentable la producción de árboles por ejidatarios en la península de Yucatán. II Foro Internacional. Los aprovechamientos forestales y su relación con el ambiente. Veracruz, México. s/p.

Huang, S., Y. Yang y Y. Wang. 2003. A critical look at procedures for validating growth and yield models. En: Amaro, A., Redd, D., Soares, P. (Eds.) Modelling Forest Systems. CABI. p: 271-293.

INEGI (Instituto Nacional de Estadística y Geografía). 2000. Marco Geoestadístico. Disponible en línea: http:// mapserver.inegi.gob.mx/geografia/esp anol/ datosgeogra/ basicos/ estados/ tab_geo.cfm. Consultada el 20 de agosto de 2009.

INEGI (Instituto Nacional de Estadística, Geografía e Informática). 2005. Cuaderno estadístico municipal de Jalapa, Tabasco. Impreso en México. 207 p.

Jerez, J., L. Vicent, Y. Moret y R. González. 2003. Regímenes de espaciamiento inicial y aclareo en plantaciones de Teca (Tectona grandis) L.F. en Venezuela. 16 p. Disponible en línea: http://www.una.ac.cr/inis/docs/teca/tem as/ RegmenesdEspaciamiento1.pdf. Consultada el 06 de agosto del 2009.

Klepac, D. 1983. Crecimiento e incremento de árboles y masas forestales. 2a. ed. Universidad Autónoma Chapingo. Chapingo, México. 365 p. 
Langsaeter, A. 1941. Om tynning i enaldret gran-og furuskog (About thinning in even-aged stands of spruce, fir and pine), Meddel. F.D. Norske Skogforsoksvesen 8:131-216.

Long, J.N. 1985. A practical approach to density management. Forestry Chronicle 61:23-27.

Lonsdale, W.M. y A.R. Watkinson. 1983. Plant geometry and sel-fthinning. Journal of Ecology 71:285-297.

Lugo, A.E., J.C. Figueroa-Colón y M. Alayón. 2003. Big-Leaf Mahogany: Genetic, Ecology, and Management. Springer Verlag. Nueva York. 433 p.

Madrigal H., S. y H. Ramírez M. 1995. Comparación de nueve modelos empíricos para la determinación del índice de sitio en Michoacán. Ciencia Forestal en México 20(78):35-57.

Mayhew, J.E. y A.C. Newton. 1998. The silviculture of mahogany (Swietenia macropylla). 1a. ed. CABI Publications. Wallingford, Reino Unido. 226 p.

Murillo B., Y. 2008. Determinación de aclareo en plantaciones de Cedro (Cedrela odorata L.) en el estado de Tabasco. Tesis de Maestría, Campus Tabasco, H. Cárdenas, Tabasco. 88 p.

Palma-López, D.J., E. Moreno C., J.A. RincónRamírez y E. D. Shirma T. 2008. Degradación y conservación de los suelos del estado de Tabasco. Colegio de Postgraduados, Conacyt, CCYTET. Villahermosa, Tabasco, México. $74 \mathrm{p}$.
Ramírez M., H. y M. Zepeda B. 1994. Rendimientos maderables de especies forestales, actualidades en México. En: Memorias de la IV Reunión Nacional de Plantaciones Forestales. SFFSINIFAP. México, D.F. p: 15-21.

Reineke, L.H. 1933. Perfecting a stand density index for even aged forests. Journal agricultural Research 46 (7):627-637.

Rodríguez S., B. 1996. Estimación del crecimiento y relaciones dasométricas de caoba (Swietenia macrophylla King.) en plantaciones forestales. Tesis de Maestría. Colegio de Postgraduados. Montecillo, México.

Smith, D.M., B.C. Larson, M.J. Kelty y P.M. Ashton. 1997. The practice of silviculture: applied forest ecology. 9a. ed. John Wiley and Sons. Nueva York. 537 p.

Vallejos B., O. 1996. Productividad y relaciones de índice de sitio con variables fisiográficas, edafoclimáticas y foliares para Tectona grandis L., Bombacopsis quinatum (Jacq) Dugand y Gmelina arborea Roxb en Costa Rica. Turrialba, CR, CATIE. 147 p.

Wescom, R.W. 1979. Silvicultural Research Division Annual Report 1978. Department of Forestry, Fiji. s/p.

Xue, L. y A. Hagihara. 2008. Growth analysis of the competition-density effect in nonself-thinning Populus deltoides and Populus and Populus $x$ euramericana plantations. Journal of Forest Research 13(4):241-248.

Artículo recibido el 27 de noviembre de 2009.

Aceptado el 17 de febrero de 2012.

Este artículo se debe citar como: Pérez-González, G., M. Domínguez-Domínguez, P. Martínez-Zurimendi y J.D. Etchevers-Barra. 2012. Caracterización dasométrica e índice de sitio en plantaciones de caoba en Tabasco, México. Madera y Bosques 18(1):7-24. 